\section{Ewing's Sarcoma}

Mi-Yeoung Jo

Independent Practice, Sherman Oaks, CA, USA

\section{Synonyms}

Ewing sarcoma family tumors (ESFT); Peripheral primitive neuroectodermal tumors (pPNET)

\section{Definition}

Ewing sarcoma is named after Dr. James Ewing, who first described this tumor in the 1920s. It occurs primarily in bone or soft tissue and is most often found in the pelvis, femur, tibia, and ribs. It is one of the most common types of pediatric cancers and is usually found in children and young adults between the ages of 10 and 20. It is rare past the age of 30 . There is a slightly higher incidence in males than females. Pain is usually the earliest symptom and can be accompanied by swelling and fever. Nerve and spinal cord compression may cause additional symptoms such as numbness, tingling, incontinence, and paralysis.
This type of tumor often metastasizes to other parts of the body. It is usually malignant with unfavorable prognosis, particularly in those with metastatic disease. Treatment often involves whole-body chemotherapy, localized surgery, and/or radiotherapy. In children, possible late delayed effects of radiotherapy may include slowed bone growth and infertility.

\section{Cross-References}

- Late-Delayed Effects of Radiation

- Metastasis

- Neoplasm

- Primitive Neuroectodermal Tumor

$\checkmark$ Radiotherapy

Sarcoma

\section{References and Readings}

Cote, G. M., \& Choy, E. (2013). Update in treatment and targets in Ewing sarcoma. Hematology/Oncology Clinics of North America, 27(5), 1007-1019.

Ewing, J. (1921). Diffuse endothelioma of bone. Proceedings of the New York Pathological Society, 21, 17-24. 